\title{
ALEXANDER-EQUIVALENT ZARISKI PAIRS OF IRREDUCIBLE SEXTICS
}

\author{
CHRISTOPHE EYRAL AND MUTSUO OKA
}

\begin{abstract}
The existence of Alexander-equivalent Zariski pairs dealing with irreducible curves of degree 6 was proved by A. Degtyarev. However, up to now, no explicit example of such a pair was available (only the existence was known). In this paper, we construct the first concrete example.
\end{abstract}

\section{INTRODUCTION}

Let $\mathcal{M}(\Sigma, d)$ be the moduli space of reduced curves of degree $d$ in $\mathbb{C P}^{2}$ with a prescribed configuration of singularities $\Sigma$ A pair of curves $\left(C, C^{\prime}\right)$ in $\mathcal{M}(\Sigma, d)$ is said to be a Zariski pair if it satisfies the following two conditions (cf. [1]):

(1) $C$ and $C^{\prime}$ have the same combinatoric, that is, there exist regular neighbourhoods $T(C)$ and $T\left(C^{\prime}\right)$ of $C$ and $C^{\prime}$ respectively such that the pairs $(T(C), C)$ and $\left(T\left(C^{\prime}\right), C^{\prime}\right)$ are homeomorphic;

(2) the pairs $\left(\mathbb{C P}^{2}, C\right)$ and $\left(\mathbb{C P}^{2}, C^{\prime}\right)$ are not homeomorphic.

It is easy to check that if both $C$ and $C^{\prime}$ are irreducible then the first condition is always satisfied. The first Zariski pair appears in the works by O. Zariski [13, 14, 15] (see also [1] and [7]). The members of the pair are irreducible curves of degree 6, which is the smallest degree for which Zariski pairs exist.

The existence of a Zariski pair in a moduli space gives an information about its connected components. Indeed, if $\mathcal{M}(\Sigma, d)$ has a Zariski pair $\left(C, C^{\prime}\right)$, then $C$ and $C^{\prime}$ necessarily belong to different connected components (cf. [16, 17, 6]) - in particular, if $\mathcal{M}(\Sigma, d)$ has a Zariski pair, then it is not connected. (The converse statement is not clear. Two curves coming from two different connected components may have the same embedded topology.)

Now to check whether a given a pair of curves $C, C^{\prime} \in \mathcal{M}(\Sigma, d)$ with the same combinatoric is a Zariski pair, one can first try to calculate the generic Alexander polynomials of the curves. If these polynomials are different, then the curves do

2000 Mathematics Subject Classification. 14H30 (14H20, 14H45, 14H50).

Key words and phrases. Topology of plane curves; Fundamental group; Zariski pairs; Alexander polynomial.

${ }^{1}$ By such a moduli space we mean the quotient space $\mathcal{C}(\Sigma, d) / \operatorname{PGL}(3, \mathbb{C})$ of the space $\mathcal{C}(\Sigma, d)$ of reduced plane curves with degree $d$ and set of singularities $\Sigma$ by the 'standard' group action of $\operatorname{PGL}(3, \mathbb{C})$. 
not have the same embedded topology, and $\left(C, C^{\prime}\right)$ is a Zariski pair. However, it may happen that these polynomials are the same although $\left(C, C^{\prime}\right)$ is a Zariski pair. In this case the pair is said to be Alexander-equivalent. The first example of such a pair was given by E. Artal Bartolo and J. Carmona Ruber [2] for reducible curves of degree 7 . The first examples dealing with irreducible curves are due to the second author [8] (curves of degree 12) and [9] (curves of degree 8). In [3], A. Degtyarev proved that Alexander-equivalent Zariski pairs also appear on irreducible curves of degree 6 . However he did not give any explicit example (only the existence is proved). The aim of the present paper is to construct a concrete example of such a pair.

\section{Statement of the Result}

Let $(X: Y: Z)$ be homogeneous coordinates on $\mathbb{C P}^{2}$ and $(x, y)$ the affine coordinates defined by $x:=X / Z$ and $y:=Y / Z$ on $\mathbb{C P}^{2} \backslash\{Z=0\}$, as usual. We consider the projective curves $C$ and $C^{\prime}$ in $\mathbb{C P}^{2}$ defined by the affine equations $f(x, y)=0$ and $f^{\prime}(x, y)=0$ respectively, where

$$
\begin{aligned}
f(x, y):= & \frac{369}{364} y^{6}+y^{5} x-\frac{197}{91} y^{5}+\frac{207}{182} y^{4} x^{2}-\frac{185}{91} y^{4} x+\frac{235}{182} y^{4}+ \\
& \frac{87}{182} y^{3} x^{3}-\frac{255}{182} y^{3} x^{2}+\frac{97}{91} y^{3} x-\frac{1}{7} y^{3}+\frac{101}{364} y^{2} x^{4}-\frac{47}{91} y^{2} x^{3}+ \\
& \frac{7}{26} y^{2} x^{2}-\frac{3}{91} y^{2} x+\frac{1}{364} y^{2}+\frac{5}{182} y x^{5}-\frac{11}{182} y x^{4}+\frac{1}{26} y x^{3}- \\
& \frac{1}{182} y x^{2}+\frac{1}{364} x^{6}-\frac{1}{182} x^{5}+\frac{1}{364} x^{4}, \\
f^{\prime}(x, y):= & -\frac{4}{3} y^{6}+\left(-\frac{8}{9} x^{2}+4 x+1\right) y^{4}+\left(-\frac{4}{9} x^{4}+\frac{26}{9} x^{3}-\frac{14}{3} x^{2}-2 x\right) y^{2} \\
& +\frac{1}{9} x^{6}+\frac{2}{9} x^{5}-\frac{17}{9} x^{4}+2 x^{3}+x^{2} .
\end{aligned}
$$

Both $C$ and $C^{\prime}$ are irreducible sextics with the set of singularities $\mathbf{A}_{9} \oplus 2 \mathbf{A}_{4}$. (We recall that a point $P$ in a curve $D$ is said to be an $\mathbf{A}_{n}$-singularity $(n \geq 1)$ if the germ $(D, P)$ is topologically equivalent to the germ at the origin of the curve defined by $x^{2}+y^{n+1}=0$.) For the curve $C$, the $\mathbf{A}_{4}$-singularities are located at $(1,0)$ and $(0,1)$ while the $\mathbf{A}_{9}$-singularity is at $(0,0)$. For $C^{\prime}$, the $\mathbf{A}_{4}$-singularities are at $(1, \pm 1)$ and the $\mathbf{A}_{9}$-singularity is at $(0,0)$. The real plane sections of $C$ and $C^{\prime}$ are shown in Figure 1 and 2 respectively. (In the figures we do not respect the numerical scale.) The curve $C^{\prime}$ is symmetric with respect to the $x$-axis. The curve $C$ has no particular symmetry. Notice that after the analytic change of coordinates

$$
(x, y) \mapsto\left(x-\frac{1}{3} y^{4}+y^{2}, y\right)
$$


the equation of $C^{\prime}$ near the origin takes the form

$$
x^{2}+\frac{4}{27} y^{10}+\text { higher terms }=0 .
$$

As the leading term $x^{2}+(4 / 27) y^{10}$ is positive on $\mathbb{R}^{2} \backslash\{(0,0)\}$, the origin is an isolated point of the real plane section of $C^{\prime}$ (cf. Figure 2). Finally, since $C$ and $C^{\prime}$ are not of torus type (cf. [10] and [11]), their generic Alexander polynomials are trivial (cf. [3]).

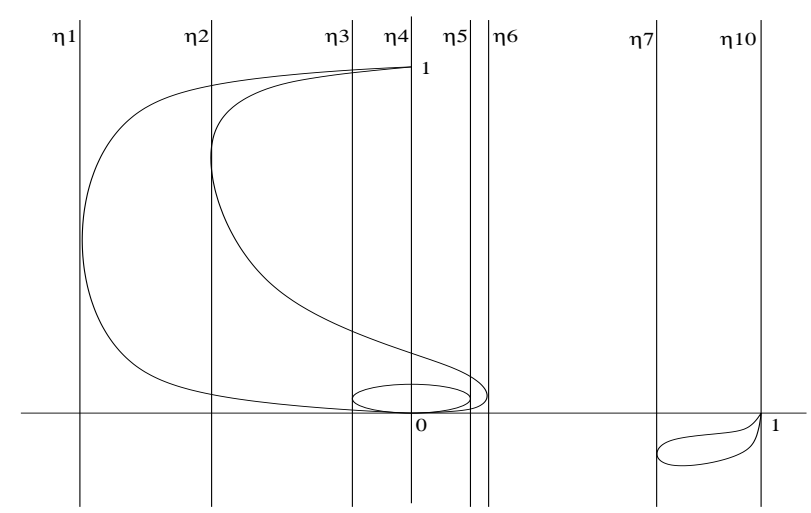

Figure 1. $\left\{(x, y) \in \mathbb{R}^{2} ; f(x, y)=0\right\}$

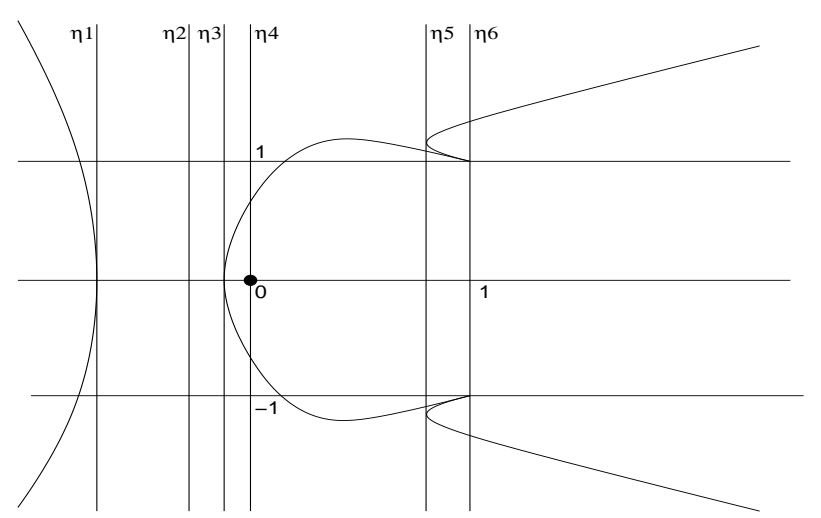

Figure 2. $\left\{(x, y) \in \mathbb{R}^{2} ; f^{\prime}(x, y)=0\right\}$

Theorem 2.1. The fundamental group $\pi_{1}\left(\mathbb{C P}^{2} \backslash C\right)$ is isomorphic to $\mathbb{Z} / 6 \mathbb{Z}$ while $\pi_{1}\left(\mathbb{C P}^{2} \backslash C^{\prime}\right)$ is isomorphic to $\mathbb{D}_{10} \times(\mathbb{Z} / 3 \mathbb{Z})$, where $\mathbb{D}_{10}$ is the dihedral group of order 10. In particular $\left(C, C^{\prime}\right)$ is an Alexander-equivalent Zariski pair and the 
moduli space of irreducible sextics with the set of singularities $\mathbf{A}_{9} \oplus 2 \mathbf{A}_{4}$ has at least two connected components?

Theorem 2.1 is proved in sections 3 and 4 below

The curve $C^{\prime}$ is an example of so-called $\mathbb{D}_{10}$-sextics. ( $\mathrm{A} \mathbb{D}_{10}$-sextic is a nontorus irreducible sextic with simple singularities and whose fundamental group 3 factors to the dihedral group $\mathbb{D}_{10}$.) The existence of $\mathbb{D}_{10}$-sextics was first proved, purely arithmetically, by A. Degtyarev [3] who showed that there exist exactly 8 equisingular deformation families of such curves, one family for each of the following sets of singularities:

$$
4 \mathbf{A}_{4}, 4 \mathbf{A}_{4} \oplus \mathbf{A}_{1}, 4 \mathbf{A}_{4} \oplus 2 \mathbf{A}_{1}, 4 \mathbf{A}_{4} \oplus \mathbf{A}_{2},
$$

$$
\mathbf{A}_{9} \oplus 2 \mathbf{A}_{4}, \quad \mathbf{A}_{9} \oplus 2 \mathbf{A}_{4} \oplus \mathbf{A}_{1}, \quad \mathbf{A}_{9} \oplus 2 \mathbf{A}_{4} \oplus \mathbf{A}_{2}, 2 \mathbf{A}_{9} .
$$

First explicit examples and fundamental groups of $\mathbb{D}_{10}$-sextics were given in [4] (see also [5] for the sets of singularities $4 \mathbf{A}_{4}$ and $4 \mathbf{A}_{4} \oplus \mathbf{A}_{1}$ ).

Furthermore, Degtyarev also observed in [3] that the configurations

$$
4 \mathbf{A}_{4}, 4 \mathbf{A}_{4} \oplus \mathbf{A}_{1}, \quad \mathbf{A}_{9} \oplus 2 \mathbf{A}_{4}, \quad \mathbf{A}_{9} \oplus 2 \mathbf{A}_{4} \oplus \mathbf{A}_{1}, 2 \mathbf{A}_{9}
$$

can be realized not only by $\mathbb{D}_{10}$-sextics but also by irreducible non-torus sextics $D$ for which the group $\pi_{1}\left(\mathbb{C P}^{2} \backslash D\right)$ does not factors to $\mathbb{D}_{10}$. (In particular, since the Alexander polynomial of an irreducible non-torus sextic with simple singularities is always trivial, these 5 sets of singularities give rise to Alexanderequivalent Zariski pairs of irreducible sextics.) However, Degtyarev did not give any explicit equation of such a curve $D$ (only the existence is proved) and did not calculate the group $\pi_{1}\left(\mathbb{C P}^{2} \backslash D\right)$. The curve $C$ in Theorem 2.1 is the first explicit example of such a curve - i.e., a curve whose fundamental group does not admit a dihedral quotient although its set of singularities can be realized by a $\mathbb{D}_{10}$-sextic as well. (In particular, the pair $\left(C, C^{\prime}\right)$ in Theorem 2.1 is also the first concrete example of an Alexander-equivalent Zariski pair dealing with irreducible sextics.)

\section{Fundamental group of $\mathbb{C P}^{2} \backslash C$}

In this section, we show that $\pi_{1}\left(\mathbb{C P}^{2} \backslash C\right) \simeq \mathbb{Z} / 6 \mathbb{Z}$. In fact, it suffices to prove that $\pi_{1}\left(\mathbb{C P}^{2} \backslash C\right)$ is abelian. Indeed, by Hurewicz's theorem, if $\pi_{1}\left(\mathbb{C P}^{2} \backslash C\right)$ is abelian, then it is isomorphic to first integral homology group $H_{1}\left(\mathbb{C P}^{2} \backslash C\right)$ and it is well known that $H_{1}\left(\mathbb{C P}^{2} \backslash C\right) \simeq \mathbb{Z} / 6 \mathbb{Z}$.

To show that $\pi_{1}\left(\mathbb{C P}^{2} \backslash C\right)$ is abelian, we use Zariski-van Kampen's theorem with the pencil given by the vertical lines $L_{\eta}: x=\eta, \eta \in \mathbb{C}$ (cf. [13] and [12]). We always take the point $(0: 1: 0)$ as base point for our fundamental groups.

\footnotetext{
${ }^{2}$ Though the existence of the structure of an algebraic variety on a moduli space is not always obvious, the moduli space we consider here has such a structure. The last assertion in the theorem then implies this moduli space has at least two irreducible components as well.

${ }^{3}$ We always mean the fundamental group of the complement of the curve.
} 
This point is nothing but the axis of the pencil, which is also the point at infinity of the lines $L_{\eta}$. Note that it does not belong to the curve.

The discriminant $\Delta_{y}(f)$ of $f$ as a polynomial in $y$ is the polynomial in $x$ given by

$$
\begin{array}{r}
\Delta_{y}(f)(x)=a_{0} x^{15}(x-1)^{7}\left(858898351 x^{8}-1278576626 x^{7}-359900737 x^{6}+\right. \\
\left.1017975356 x^{5}-56181608 x^{4}-170653568 x^{3}+2388080 x^{2}+2072000 x-96000\right),
\end{array}
$$

where $a_{0} \in \mathbb{Q} \backslash\{0\}$. This polynomial has exactly 10 distinct complex roots:

$$
\begin{aligned}
& \eta_{1} \approx-0.7408, \quad \eta_{2} \approx-0.3914, \quad \eta_{3} \approx-0.1309, \quad \eta_{4}=0, \\
& \eta_{5} \approx 0.0598, \quad \eta_{6} \approx 0.0778, \quad \eta_{7} \approx 0.6274, \\
& \eta_{8} \approx 0.9933-i 0.1446, \quad \eta_{9}=\bar{\eta}_{8} \approx 0.9933+i 0.1446, \quad \eta_{10}=1 .
\end{aligned}
$$

The singular lines of the pencil are the lines $L_{\eta_{j}}(1 \leq j \leq 10)$ corresponding to these 10 roots. The lines $L_{\eta_{4}}$ and $L_{\eta_{10}}$ intersect the curve at its singular points. All the other singular lines are tangent to $C$. See Figure 1 .

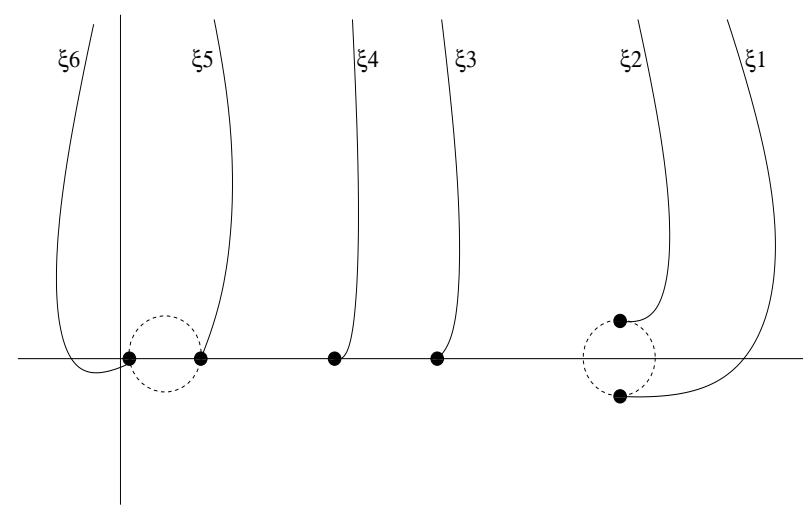

FiguRE 3. Generators at $x=\eta_{4}+\varepsilon$

We consider the generic line $L_{\eta_{4}+\varepsilon}$ and choose generators $\xi_{1}, \ldots, \xi_{6}$ of the fundamental group $\pi_{1}\left(L_{\eta_{4}+\varepsilon} \backslash C\right)$ as in Figure 3 , where $\varepsilon>0$ is small enough. The $\xi_{k}$ 's $(1 \leq k \leq 6)$ are lassos oriented counter-clockwise around the 6 intersection points of the line $L_{\eta_{4}+\varepsilon}$ with the curve - i.e., the 6 complex roots of the equation $f\left(\eta_{4}+\varepsilon, y\right)=0$. (In the figures, a lasso is represented by a path ending with a bullet.) The Zariski-van Kampen theorem says that

$$
\pi_{1}\left(\mathbb{C P}^{2} \backslash C\right) \simeq \pi_{1}\left(L_{\eta_{4}+\varepsilon} \backslash C\right) / G,
$$

where $G$ is the normal subgroup of $\pi_{1}\left(L_{\eta_{4}+\varepsilon} \backslash C\right)$ generated by the monodromy relations associated with the singular lines of the pencil. To find these relations, we fix a 'standard' system of generators $\sigma_{1}, \ldots, \sigma_{10}$ for the fundamental group $\pi_{1}\left(\mathbb{C} \backslash\left\{\eta_{1}, \ldots, \eta_{10}\right\}\right)$ as follows. Each $\sigma_{j}$ is a lasso (oriented counter-clockwise) around $\eta_{j}$ with base point $\eta_{4}+\varepsilon$. For $j \neq 8,9$, the tail of $\sigma_{j}$ is a union of real 
segments and half-circles around the exceptional parameters $\eta_{l}(l \neq j)$ located in the real axis between the base point $\eta_{4}+\varepsilon$ and $\eta_{j}$. Its head is the circle $\mathbb{S}_{\varepsilon}\left(\eta_{j}\right)$ with centre $\eta_{j}$ and radius $\varepsilon$. The lasso $\sigma_{8}$ corresponding to the non-real root $\eta_{8}$ is given by $\zeta \theta \zeta^{-1}$, where $\theta$ is the loop obtained by moving $x$ once on the circle $\mathbb{S}_{\varepsilon}\left(\eta_{8}\right)$, starting at $\Re\left(\eta_{8}\right)+i\left(\Im\left(\eta_{8}\right)+\varepsilon\right)$, while $\zeta$ is the path obtained when $x$ moves on the real axis from $\eta_{4}+\varepsilon$ to $\eta_{5}-\varepsilon$, makes half-turn on the circle $\mathbb{S}_{\varepsilon}\left(\eta_{5}\right)$, from $\eta_{5}-\varepsilon$ to $\eta_{5}+\varepsilon$, moves on the real axis from $\eta_{5}+\varepsilon$ to $\eta_{6}-\varepsilon$, makes half-turn on the circle $\mathbb{S}_{\varepsilon}\left(\eta_{6}\right)$, from $\eta_{6}-\varepsilon$ to $\eta_{6}+\varepsilon$, moves on the real axis from $\eta_{6}+\varepsilon$ to $\eta_{7}-\varepsilon$, makes half-turn on the circle $\mathbb{S}_{\varepsilon}\left(\eta_{7}\right)$, from $\eta_{7}-\varepsilon$ to $\eta_{7}+\varepsilon$, moves on the real axis from $\eta_{7}+\varepsilon$ to $\Re\left(\eta_{8}\right)$, and finally moves in a straight line from $\Re\left(\eta_{8}\right)$ to $\Re\left(\eta_{8}\right)+i\left(\Im\left(\eta_{8}\right)+\varepsilon\right)$. (Here $\Re\left(\eta_{8}\right)$ and $\Im\left(\eta_{8}\right)$ denote the real and the imaginary parts of $\eta_{8}$ respectively.) The lasso $\sigma_{9}$ is defined similarly from a loop $\theta$ and a path $\zeta$ meeting at $\Re\left(\eta_{9}\right)+i\left(\Im\left(\eta_{9}\right)-\varepsilon\right)$. The monodromy relations around the singular line $L_{\eta_{j}}$ are obtained by moving the generic 'fibre' $F \simeq L_{\eta_{4}+\varepsilon} \backslash C$ isotopically 'above' the loop $\sigma_{j}$, and by identifying each $\xi_{k}(1 \leq k \leq 6)$ with its image by the terminal homeomorphism of this isotopy. (For details see [13] and [12].)

The remaining of the proof is to determine these relations.

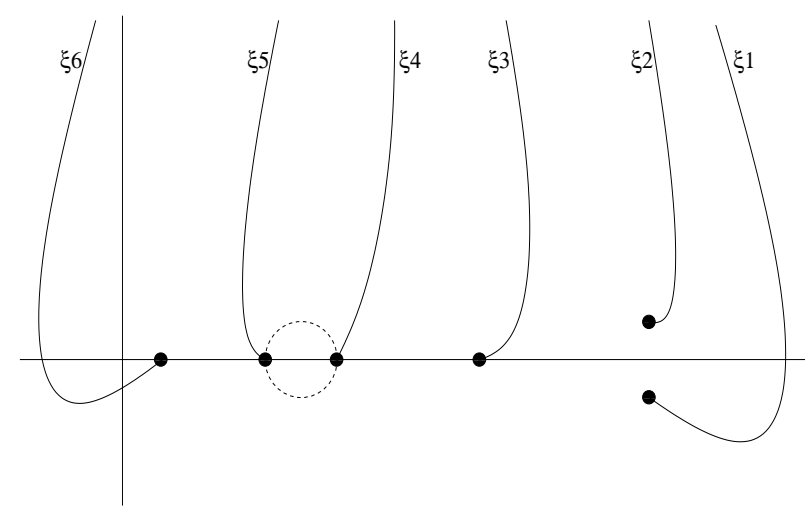

Figure 4. Generators at $x=\eta_{5}-\varepsilon$

Monodromy relations at $x=\eta_{5}$. When $x$ moves on the real axis from $\eta_{4}+\varepsilon$ to $\eta_{5}-\varepsilon$, the 6 complex roots of the equation (in $y$ ) $f(x, y)=0$ (and, consequently, the 6 generators $\left.\xi_{1}, \ldots \xi_{6}\right)$ are deformed as in Figure 4 . The singular line $L_{\eta_{5}}$ is tangent to the curve at the simple point $P \approx\left(\eta_{5}, 0.0095\right)$, and the intersection multiplicity $I\left(L_{\eta_{5}}, C ; P\right)$ of this line with the curve at this point is 2 . Therefore, by the implicit functions theorem, the germ $(C, P)$ is given by

$$
x-\eta_{5}=b_{0}(y-0.0095)^{2}+\text { higher terms, }
$$

where $b_{0} \neq 0$. It follows that when $x$ runs once counter-clockwise on the circle $\mathbb{S}_{\varepsilon}\left(\eta_{5}\right)$, starting at $\eta_{5}-\varepsilon$, the variable $y$ makes half-turn counter-clockwise on the 
dotted circle around 0.0095 (cf. Figure 4). The monodromy relation at $x=\eta_{5}$ is then given by

$$
\xi_{5}=\xi_{4}
$$

Monodromy relations at $x=\eta_{6}$. At $x=\eta_{5}-\varepsilon$, the generators are as in Figure 4. In Figure 5, we show how the $\xi_{k}$ 's are deformed when $x$ first makes half-turn counter-clockwise on the circle $\mathbb{S}_{\varepsilon}\left(\eta_{5}\right)$, from $\eta_{5}-\varepsilon$ to $\eta_{5}+\varepsilon$, and then moves on the real axis from $\eta_{5}+\varepsilon$ to $\eta_{6}-\varepsilon$. The singular line $L_{\eta_{6}}$ is also tangent to $C$ at a simple point $P^{\prime}$ and $I\left(L_{\eta_{6}}, C ; P^{\prime}\right)=2$. Therefore, as above, the monodromy relation we are looking for is simply given by

$$
\xi_{3}=\xi_{4}^{-1} \xi_{6} \xi_{4} \text {. }
$$

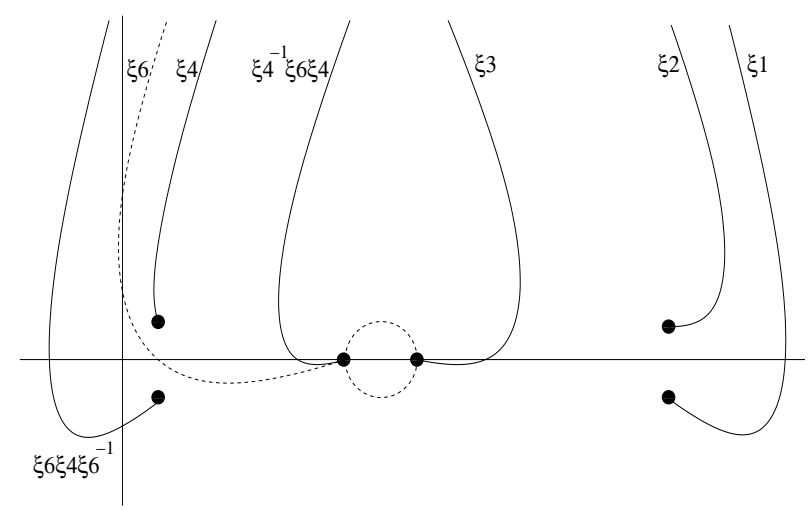

FiguRE 5. Generators at $x=\eta_{6}-\varepsilon$

Monodromy relations at $x=\eta_{4}$. The singular line $L_{\eta_{4}}$ passes through the singular points $(0,0)$ and $(0,1)$ which are singularities of type $\mathbf{A}_{9}$ and $\mathbf{A}_{4}$ repectively. At $(0,0)$, the curve has two branches $K_{-}$and $K_{+}$given by

$$
K_{ \pm}: \quad y=x^{2}+5 x^{3}+51 x^{4}+(503 \pm 32 \sqrt{6}) x^{5}+\text { higher terms. }
$$

It follows that when $x$ runs once counter-clockwise on the circle $\mathbb{S}_{\varepsilon}\left(\eta_{4}\right)$, starting at $\eta_{4}+\varepsilon$, the generators $\xi_{6}$ and $\xi_{5}=\xi_{4}$ make 5 turns counter-clockwise on the corresponding dotted circle (cf. Figure 3). The monodromy relation around $L_{\eta_{4}}$ that comes from the singular point $(0,0)$ is then given by

$$
\xi_{4}=\left(\xi_{6} \xi_{4}\right)^{5} \cdot \xi_{4} \cdot\left(\xi_{6} \xi_{4}\right)^{-5} .
$$

At $(0,1)$, a Puiseux parametrization of $C$ is given by

$$
x=t^{2}, \quad y=1-\frac{1}{2} t^{4}+\frac{1}{10} i \sqrt{5} t^{5}+\text { higher terms. }
$$

Hence, when $x$ goes once counter-clockwise on the circle $\mathbb{S}_{\varepsilon}\left(\eta_{4}\right)$, starting at $\eta_{4}+\varepsilon$, the generators $\xi_{1}$ and $\xi_{2}$ make (5/2)-turn counter-clockwise on the corresponding 
dotted circle (cf. Figure 3). The monodromy relation around $L_{\eta_{4}}$ that comes from the singular point $(0,1)$ is then given by

$$
\xi_{1}=\left(\xi_{2} \xi_{1}\right)^{2} \cdot \xi_{2} \cdot\left(\xi_{2} \xi_{1}\right)^{-2} .
$$

Monodromy relations at $x=\eta_{3}$. At $x=\eta_{4}+\varepsilon$, the generators are as in Figure 3. Now, when $x$ makes half-turn counter-clockwise on the circle $\mathbb{S}_{\varepsilon}\left(\eta_{4}\right)$, from $\eta_{4}+\varepsilon$ to from $\eta_{4}-\varepsilon$, and then moves on the real axis from $\eta_{4}-\varepsilon$ to $\eta_{3}+\varepsilon$, the $\xi_{k}$ 's are deformed as in Figure 6, where

$$
\gamma:=\left(\xi_{6} \xi_{4}\right)^{-2} \cdot \xi_{4}^{-1} \xi_{6} \xi_{4} \cdot\left(\xi_{6} \xi_{4}\right)^{2} .
$$

The singular line $L_{\eta_{3}}$ is tangent to the curve at a simple point, with intersection multiplicity 2, and the monodromy relation we are looking for is given by

$$
\xi_{4}=\gamma
$$

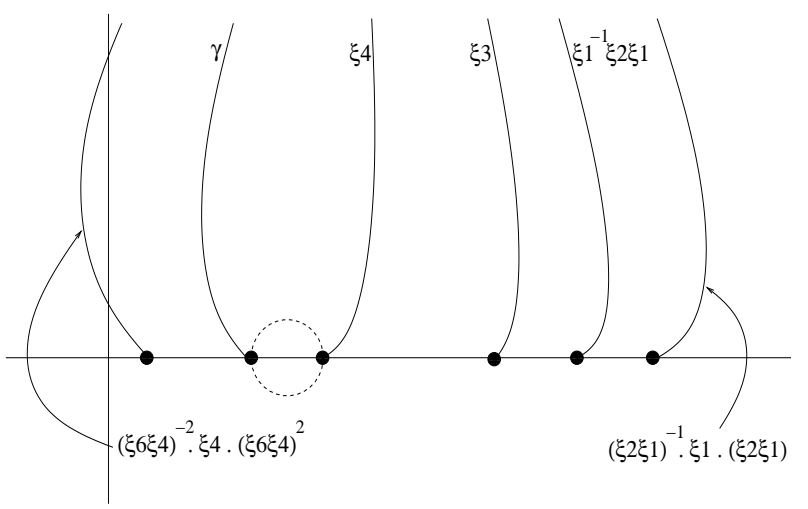

FiguRE 6 . Generators at $x=\eta_{3}+\varepsilon$

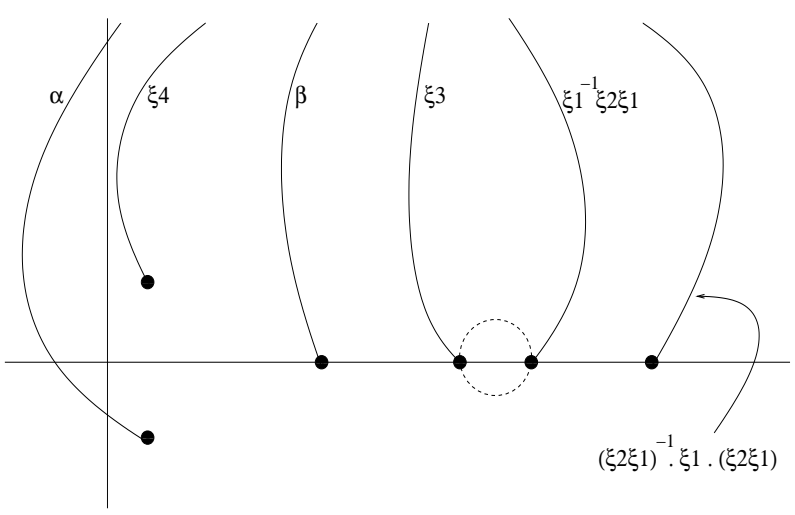

FiguRE 7 . Generators at $x=\eta_{2}+\varepsilon$ 
Monodromy relations at $x=\eta_{2}$. When $x$ makes half-turn counter-clockwise on the circle $\mathbb{S}_{\varepsilon}\left(\eta_{3}\right)$, from $\eta_{3}+\varepsilon$ to $\eta_{3}-\varepsilon$, then moves on the real axis from $\eta_{3}-\varepsilon$ to $\eta_{2}+\varepsilon$, the $\xi_{k}$ 's are deformed as in Figure 7, where

$$
\begin{aligned}
\alpha & :=\left(\left(\xi_{6} \xi_{4}\right)^{-2} \cdot \xi_{4} \cdot\left(\xi_{6} \xi_{4}\right)^{2}\right) \cdot \xi_{4} \cdot\left(\left(\xi_{6} \xi_{4}\right)^{-2} \cdot \xi_{4} \cdot\left(\xi_{6} \xi_{4}\right)^{2}\right)^{-1}, \\
\beta & :=\xi_{4}^{-1} \cdot\left(\xi_{6} \xi_{4}\right)^{-2} \cdot \xi_{4} \cdot\left(\xi_{6} \xi_{4}\right)^{2} \cdot \xi_{4} .
\end{aligned}
$$

The monodromy relation at $x=\eta_{2}$ is also an usual multiplicity 2 tangent relation:

$$
\xi_{3}=\xi_{1}^{-1} \xi_{2} \xi_{1} \text {. }
$$

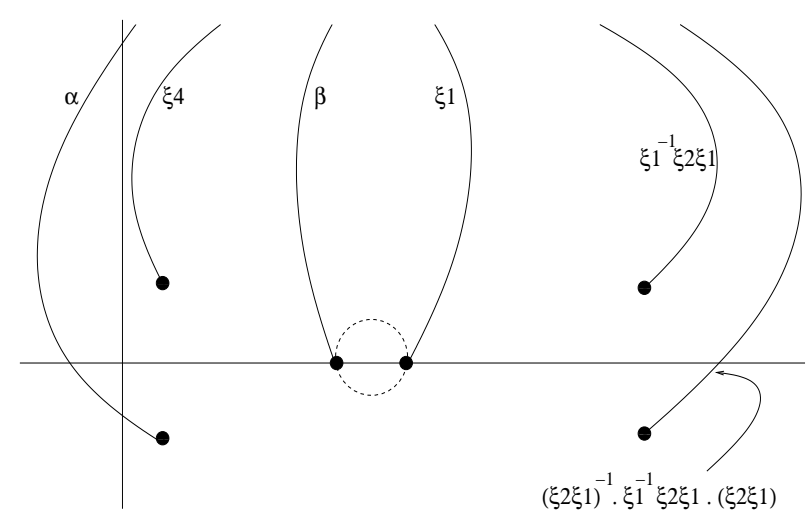

FiguRE 8. Generators at $x=\eta_{1}+\varepsilon$

Monodromy relations at $x=\eta_{1}$. In Figure 8 , we show how the $\xi_{k}$ 's are deformed when $x$ makes half-turn counter-clockwise on the circle $\mathbb{S}_{\varepsilon}\left(\eta_{2}\right)$, from $\eta_{2}+\varepsilon$ to $\eta_{2}-\varepsilon$, then moves on the real axis from $\eta_{2}-\varepsilon$ to $\eta_{1}+\varepsilon$. The monodromy relation around $L_{\eta_{1}}$ is a multiplicity 2 tangent relation given by

$$
\xi_{1}=\beta \text {. }
$$

Equivalently $\left(\xi_{6} \xi_{4}\right)^{2} \cdot \xi_{4}=\xi_{4}^{-1}\left(\xi_{6} \xi_{4}\right)^{2} \cdot \xi_{4} \xi_{1}$. Since $\left(\xi_{6} \xi_{4}\right)^{2} \cdot \xi_{4}=\xi_{4}^{-1}\left(\xi_{6} \xi_{4}\right)^{2} \cdot \xi_{6} \xi_{4}$ (by (3.5)), it follows that

$$
\xi_{4} \xi_{1}=\xi_{6} \xi_{4}
$$

Combined with (3.2), this gives

$$
\xi_{3}=\xi_{1}
$$

Combined with (3.6), this in turn implies

$$
\xi_{2}=\xi_{1} .
$$


Monodromy relations at $x=\eta_{7}$. We recall that, at $x=\eta_{6}-\varepsilon$, the generators are as in Figure 5. When $x$ makes half-turn counter-clockwise on the circle $\mathbb{S}_{\varepsilon}\left(\eta_{6}\right)$, from $\eta_{6}-\varepsilon$ to $\eta_{6}+\varepsilon$, the $\xi_{k}$ 's are deformed as shown in Figure 9, where $\delta:=\left(\xi_{6} \xi_{4} \xi_{6}^{-1} \xi_{4}\right) \cdot \xi_{1} \cdot\left(\xi_{6} \xi_{4} \xi_{6}^{-1} \xi_{4}\right)^{-1}$.

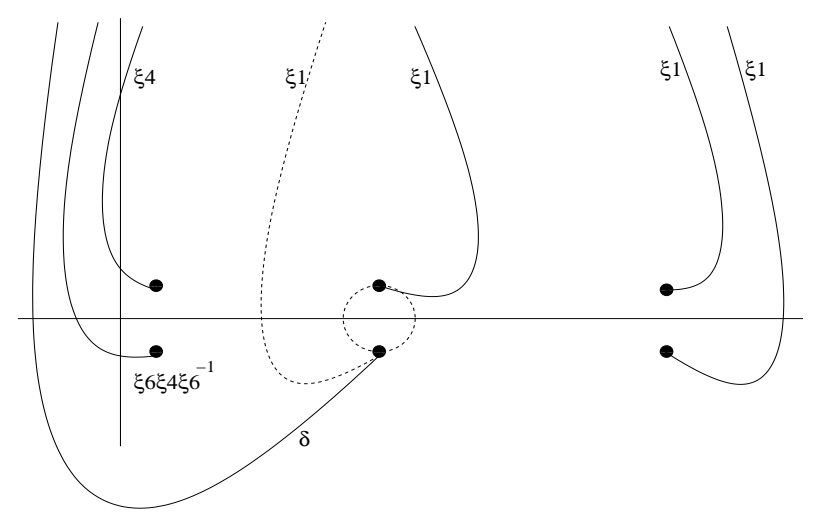

Figure 9. Generators at $x=\eta_{6}+\varepsilon$

Lemma 3.1. When $x$ moves on the real axis from $\eta_{6}+\varepsilon$ to $\eta_{7}-\varepsilon$, the $\xi_{k}$ 's are deformed as Figure 10.

Lemma 3.1 is not obvious and will be proved at the end of this section. Before, let us complete the calculation of $\pi_{1}\left(\mathbb{C P}^{2} \backslash C\right)$.

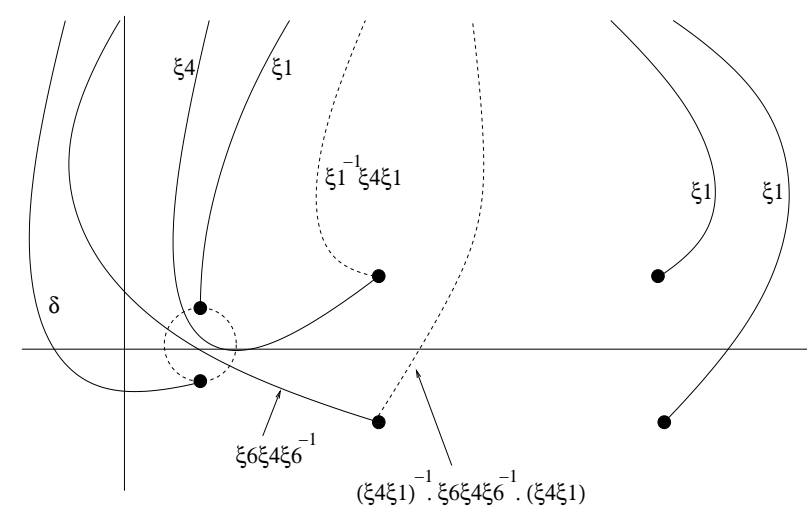

Figure 10. Generators at $x=\eta_{7}-\varepsilon$

The monodromy relation at $x=\eta_{7}$ is a multiplicity 2 tangent relation given by

$$
\delta=\xi_{1} \text {. }
$$

Notice that, by (3.8), this relation can also be written as

$$
\xi_{4} \xi_{1} \xi_{4} \xi_{1}=\xi_{1} \xi_{4} \xi_{1} \xi_{4}
$$


Moreover, still using (3.8), we can write the relation (3.3) as

$$
\xi_{4}=\left(\xi_{4} \xi_{1}\right)^{5} \cdot \xi_{4} \cdot\left(\xi_{4} \xi_{1}\right)^{-5} .
$$

The latter, combined with (3.12), implies

$$
\xi_{4} \xi_{1}=\xi_{1} \xi_{4} .
$$

This already shows that the fundamental group $\pi_{1}\left(\mathbb{C P}^{2} \backslash C\right)$ is abelian. (We do not need to consider the monodromy relations around $L_{\eta_{8}}, L_{\eta_{9}}$ and $L_{\eta_{10}}$.)

To complete the calculation it remains to prove Lemma 3.1 .

Proof of Lemma 3.1. We consider the polynomial

$$
h(x, u, v):=f(x, u+i v)
$$

for $x, u$ and $v$ real. We denote by $f_{e}(x, u, v)$ and $f_{o}(x, u, v)$ the real and the imaginary part of $h(x, u, v)$ respectively. They have degree 6 and 5 respectively in $v$. Suppose there exists a number $x_{0} \in\left[\eta_{6}+\varepsilon, \eta_{7}-\varepsilon\right]$ such that 4 complex solutions of the equation $f\left(x_{0}, y\right)=0$ are on the same vertical line $u=u_{0}$ in the complex plane $(\mathbb{C}, y=u+i v)$. This implies that the equations

$$
f_{e}\left(x_{0}, u_{0}, v\right)=f_{o}\left(x_{0}, u_{0}, v\right)=0
$$

have 4 common real solutions $v_{1}, v_{2}, v_{3}$ and $v_{4}$. These solutions are non-zero since the equation $\Delta_{y}(f)(x)=0$ does not have any solution in $\left[\eta_{6}+\varepsilon, \eta_{7}-\varepsilon\right]$. Therefore, the equations

$$
f_{e}\left(x_{0}, u_{0}, v\right)=f_{o o}\left(x_{0}, u_{0}, v\right)=0,
$$

where $f_{o o}(x, u, v)=f_{o}(x, u, v) / v$ (notice that $v$ divides $f_{o}(x, u, v)$, and $f_{o o}(x, u, v)$ is then a polynomial), also have $v_{1}, v_{2}, v_{3}$ and $v_{4}$ as common solutions. As $f_{o o}$ has degree 4 in $v$, it follows that $f_{o o}\left(x_{0}, u_{0}, v\right)$ divides $f_{e}\left(x_{0}, u_{0}, v\right)$. Therefore the remainder $R(x, u, v)$ of $f_{e}$ by $f_{o o}$, as a polynomial in $v$, must be identically zero for $u=u_{0}$ and $x=x_{0}$ (of course, $R$ is written as $R=R^{\prime} / R^{\prime \prime}$ where $R^{\prime}$ is a polynomial in $x, u$ and $v$ while $R^{\prime \prime}$ is a polynomial depending just on $x$ and $u$ ). By an easy computation, we see that $R=\left(R_{2}^{\prime} / R_{2}^{\prime \prime}\right) v^{2}+\left(R_{0}^{\prime} / R_{0}^{\prime \prime}\right)$, where $R_{2}^{\prime}, R_{2}^{\prime \prime}$, $R_{0}^{\prime}$ and $R_{0}^{\prime \prime}$ are polynomials in $x$ and $u$. Thus, $\left(x_{0}, u_{0}\right)$ is a common real solution of the equations

$$
R_{2}^{\prime}(x, u)=R_{0}^{\prime}(x, u)=0 .
$$

This implies that $x_{0}$ is a root of the resultant $\operatorname{Res}_{u}\left(R_{2}^{\prime}, R_{0}^{\prime}\right)$ of $R_{2}^{\prime}$ and $R_{0}^{\prime}$ as polynomials in $u$. Note that the condition $\operatorname{Res}_{u}\left(R_{2}^{\prime}, R_{0}^{\prime}\right)\left(x_{0}\right)=0$ is necessary to have a real partner $u_{0}$ such that $R_{2}^{\prime}\left(x_{0}, u_{0}\right)=R_{0}^{\prime}\left(x_{0}, u_{0}\right)=0$ but it is not sufficient since the possible partner $u_{0}$ might be non-real. There are 5 real solutions $x_{01}, \ldots, x_{05}$ of the equation $\operatorname{Res}_{u}\left(R_{2}^{\prime}, R_{0}^{\prime}\right)(x)=0$ in the interval $\left[\eta_{6}+\varepsilon, \eta_{7}-\varepsilon\right]$. Each of them gives a real number, say $u_{0 j}(1 \leq j \leq 5)$, such that $\left(x_{0 j}, u_{0 j}\right)$ is a solution of (3.13). Now, we have to check if these 5 solutions give 4 real roots of the polynomial $v \mapsto f_{o o}\left(x_{0}, u_{0}, v\right)$. Only the solution $\left(x_{0}, u_{0}\right): \approx(0.1205,0.0075)$ 
satisfies this requirement. Therefore we can have one (and only one) overcrossing of 4 complex roots. To check if it is the case, we look at the solutions $y$ of the equation, in $y, f(x, y)=0$ for values of $x$ near $x_{0}$. Maple actually gives an overcrossing.

\section{Fundamental group of $\mathbb{C P}^{2} \backslash C^{\prime}$}

In this section, we prove that $\pi_{1}\left(\mathbb{C P}^{2} \backslash C^{\prime}\right) \simeq \mathbb{D}_{10} \times(\mathbb{Z} / 3 \mathbb{Z})$. As above, we use Zariski-van Kampen's theorem with the pencil given by the vertical lines $L_{\eta}: x=\eta, \eta \in \mathbb{C}$.

The discriminant $\Delta_{y}(f)$ of $f$ as a polynomial in $y$ is the following polynomial in $x$ :

$$
\Delta_{y}(f)(x)=a_{0} x^{12}\left(x^{4}+2 x^{3}-17 x^{2}+18 x+9\right)\left(25 x^{2}-15 x-9\right)^{2}(x-1)^{10},
$$

where $a_{0} \in \mathbb{Q} \backslash\{0\}$. This polynomial has exactly 8 distinct complex roots:

$$
\begin{aligned}
& \eta_{1} \approx-5.5758, \quad \eta_{2} \approx-0.3708, \quad \eta_{3} \approx-0.3677, \\
& \eta_{4}=0, \quad \eta_{5} \approx 0.9708, \quad \eta_{6}=1, \\
& \eta_{7} \approx 1.9718-i 0.7077, \quad \eta_{8}=\bar{\eta}_{7} \approx 1.9718+i 0.7077 .
\end{aligned}
$$

The singular lines of the pencil are the lines $L_{\eta_{j}}(1 \leq j \leq 8)$ corresponding to these 8 roots. The lines $L_{\eta_{4}}$ and $L_{\eta_{6}}$ intersect the curve at its singular points, while all the other singular lines are tangent to the curve. See Figure 2 .

Here we start with the generic line $L_{\eta_{4}-\varepsilon}$ and we choose generators $\xi_{1}, \ldots, \xi_{6}$ of $\pi_{1}\left(L_{\eta_{4}-\varepsilon} \backslash C^{\prime}\right)$ as in Figure 11, where $\varepsilon>0$ is small enough. (To determine the position of the complex roots of the equation $f\left(\eta_{4}-\varepsilon, y\right)=0$ one may use (4.6) and (4.7) below.)

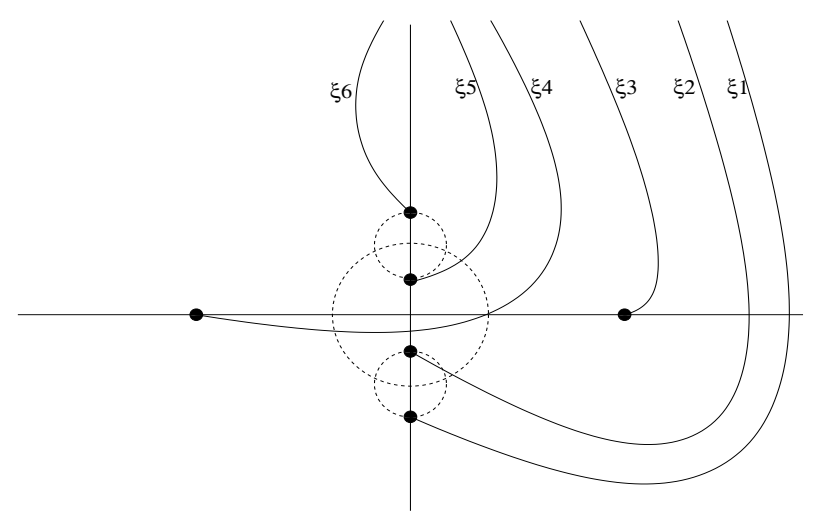

FiguRE 11. Generators at $x=\eta_{4}-\varepsilon$

As above, to find the monodromy relations around the singular lines $L_{\eta_{j}}(1 \leq$ $j \leq 8$ ) of the pencil, we fix a 'standard' system of generators $\sigma_{1}, \ldots, \sigma_{8}$ of the 
fundamental group $\pi_{1}\left(\mathbb{C} \backslash\left\{\eta_{1}, \ldots, \eta_{8}\right\}\right)$, where each $\sigma_{j}$ is a lasso (oriented counterclockwise) around $\eta_{j}$ and with base point $\eta_{4}-\varepsilon$. For $j \neq 7,8$, the tail of $\sigma_{j}$ is a union of real segments and half-circles around the exceptional parameters $\eta_{l}$ $(l \neq j)$ located in the real axis between the base point $\eta_{4}-\varepsilon$ and $\eta_{j}$, while its head is the circle $\mathbb{S}_{\varepsilon}\left(\eta_{j}\right)$. The lasso $\sigma_{7}$ corresponding to the non-real root $\eta_{7}$ has the form $\zeta \theta \zeta^{-1}$, where $\theta$ is the loop obtained by moving $x$ once on the circle $\mathbb{S}_{\varepsilon}\left(\eta_{7}\right)$, starting at $\Re\left(\eta_{7}\right)+i\left(\Im\left(\eta_{7}\right)+\varepsilon\right)$, and $\zeta$ the path obtained when $x$ makes half-turn on the circle $\mathbb{S}_{\varepsilon}\left(\eta_{4}\right)$, from $\eta_{4}-\varepsilon$ to $\eta_{4}+\varepsilon$, moves on the real axis from $\eta_{4}+\varepsilon$ to $\eta_{5}-\varepsilon$, makes half-turn on the circle $\mathbb{S}_{\varepsilon}\left(\eta_{5}\right)$, from $\eta_{5}-\varepsilon$ to $\eta_{5}+\varepsilon$, moves on the real axis from $\eta_{5}+\varepsilon$ to $\eta_{6}-\varepsilon$, makes half-turn on the circle $\mathbb{S}_{\varepsilon}\left(\eta_{6}\right)$, from $\eta_{6}-\varepsilon$ to $\eta_{6}+\varepsilon$, moves on the real axis from $\eta_{6}+\varepsilon$ to $\Re\left(\eta_{7}\right)$, and finally moves in a straight line from $\Re\left(\eta_{7}\right)$ to $\Re\left(\eta_{7}\right)+i\left(\Im\left(\eta_{7}\right)+\varepsilon\right)$. The lasso $\sigma_{8}$ is defined similarly from a loop $\theta$ and a path $\zeta$ meeting at $\Re\left(\eta_{8}\right)+i\left(\Im\left(\eta_{8}\right)-\varepsilon\right)$.

The monodromy relations are now given as follows.

Monodromy relations at $x=\eta_{3}$. When $x$ moves on the real axis from $\eta_{4}-\varepsilon$ to $\eta_{3}+\varepsilon$, the $\xi_{k}$ 's are deformed as in Figure 12. The singular line $L_{\eta_{3}}$ is tangent to $C^{\prime}$ at one simple point, with intersection multiplicity 2 , so the monodromy relation around this line is simply given by

$$
\xi_{4}=\xi_{3} .
$$

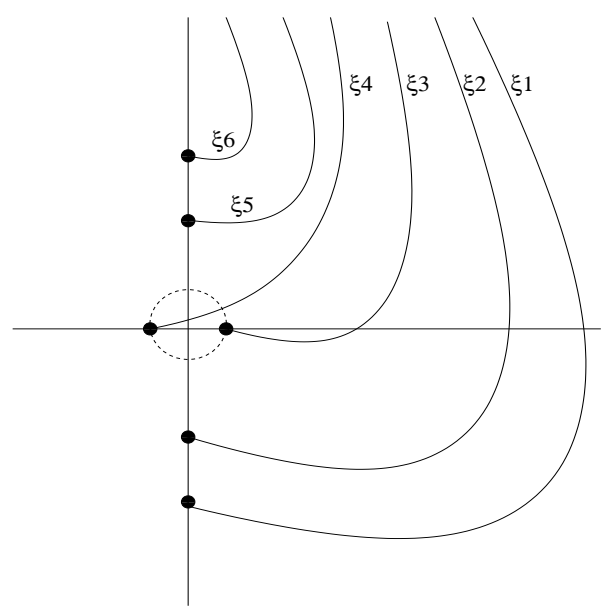

Figure 12. Generators at $x=\eta_{3}+\varepsilon$

Monodromy relations at $x=\eta_{2}$. Now, when $x$ makes half-turn counterclockwise on the circle $\mathbb{S}_{\varepsilon}\left(\eta_{3}\right)$, from $\eta_{3}+\varepsilon$ to $\eta_{3}-\varepsilon$, and then moves on the real axis from $\eta_{3}-\varepsilon$ to $\eta_{2}+\varepsilon$, the $\xi_{k}$ 's are deformed as in Figure 13. The singular line $L_{\eta_{2}}$ is tangent to the curve at two simple points, in both case with intersection 
multiplicity 2. The monodromy relations around this line are given by

$$
\begin{aligned}
& \xi_{5}=\xi_{3}, \\
& \xi_{3}=\xi_{2} .
\end{aligned}
$$

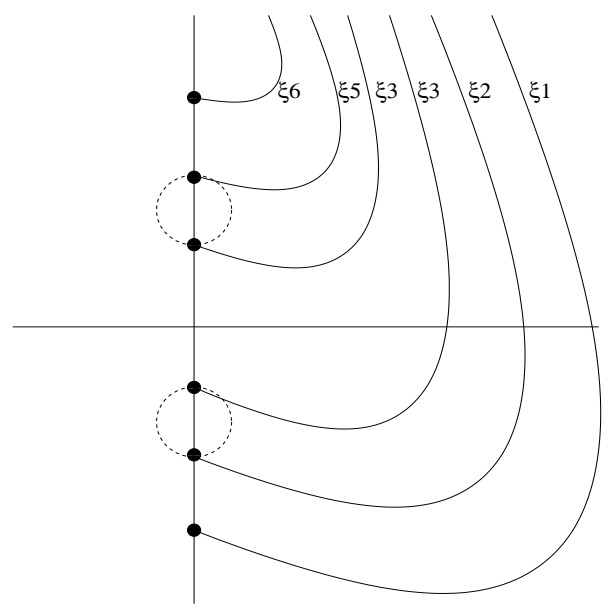

Figure 13. Generators at $x=\eta_{2}+\varepsilon$

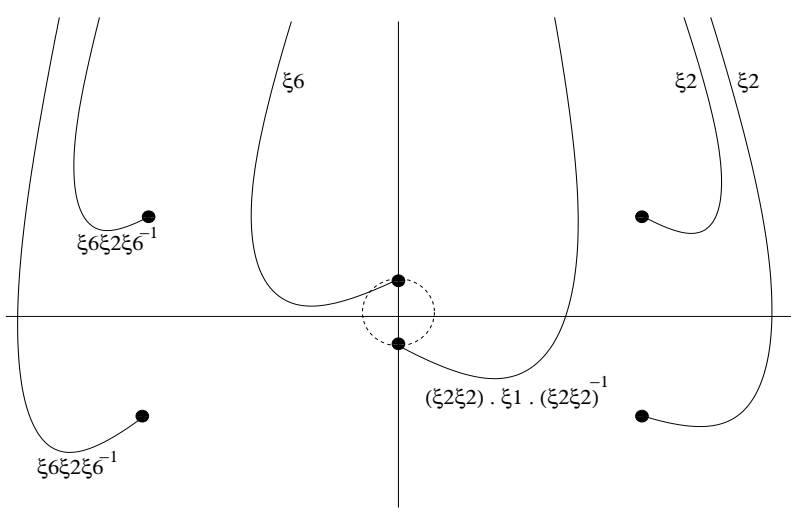

Figure 14. Generators at $x=\eta_{1}+\varepsilon$

Monodromy relations at $x=\eta_{1}$. In Figure 14, we show how the $\xi_{k}$ 's are deformed when $x$ makes half-turn counter-clockwise on the circle $\mathbb{S}_{\varepsilon}\left(\eta_{2}\right)$, from $\eta_{2}+\varepsilon$ to $\eta_{2}-\varepsilon$, and then moves on the real axis from $\eta_{2}-\varepsilon$ to $\eta_{1}+\varepsilon$. The monodromy relation around $L_{\eta_{1}}$ is a multiplicity 2 tangent relation:

$$
\xi_{6}=\xi_{2}^{2} \xi_{1} \xi_{2}^{-2}
$$


Notice that the relations (4.1)-(4.4) show that the vanishing relation at infinity can be written as

$$
\left(\xi_{2} \xi_{2} \xi_{1}\right)^{2}=e
$$

where $e$ is the unit element.

Monodromy relations at $x=\eta_{4}$. At $x=\eta_{4}-\varepsilon$, the generators are shown in Figure 11. By (4.1), (4.2) and (4.3), Figure 11 is the same as Figure 15, The singular line $L_{\eta_{4}}$ passes through the origin which a type $\mathbf{A}_{9}$ singular point of the curve. At this point the curve has two branches $K_{+}$and $K_{-}$given by

$$
K_{ \pm}: \quad x=y^{2}-\frac{1}{3} y^{4} \pm \frac{2}{9} i \sqrt{3} y^{5}+\text { higher terms. }
$$

An easy computation shows that Puiseux parametrizations of these branches are:

$$
K_{ \pm}: \quad x=t^{2}, \quad y=t+\frac{1}{6} t^{3} \mp \frac{\sqrt{3}}{9} i t^{4}+\text { higher terms. }
$$

The monodromy relations at $x=\eta_{4}$ are then given by

$$
\begin{aligned}
& \xi_{2} \xi_{1} \xi_{2}^{-1}=\left(\xi_{6} \xi_{2}\right) \cdot \xi_{6} \xi_{2} \xi_{6}^{-1} \cdot\left(\xi_{6} \xi_{2}\right)^{-1}, \\
& \xi_{2}=\left(\xi_{6} \xi_{2}\right)^{2} \cdot \xi_{6} \cdot\left(\xi_{6} \xi_{2}\right)^{-2}, \\
& \xi_{2}=\left(\xi_{6} \xi_{2}^{3} \xi_{1}\right) \cdot \xi_{2} \xi_{1} \xi_{2}^{-1} \cdot\left(\xi_{6} \xi_{2}^{3} \xi_{1}\right)^{-1}, \\
& \xi_{6}=\left(\xi_{6} \xi_{2}^{3} \xi_{1}\right) \cdot\left(\xi_{2} \xi_{1}\right) \cdot \xi_{2} \cdot\left(\xi_{2} \xi_{1}\right)^{-1} \cdot\left(\xi_{6} \xi_{2}^{3} \xi_{1}\right)^{-1} .
\end{aligned}
$$

Note that, by (4.4), all of them are equivalent to

$$
\xi_{1} \xi_{2} \xi_{1} \xi_{2} \xi_{1}=\xi_{2} \xi_{1} \xi_{2} \xi_{1} \xi_{2}
$$

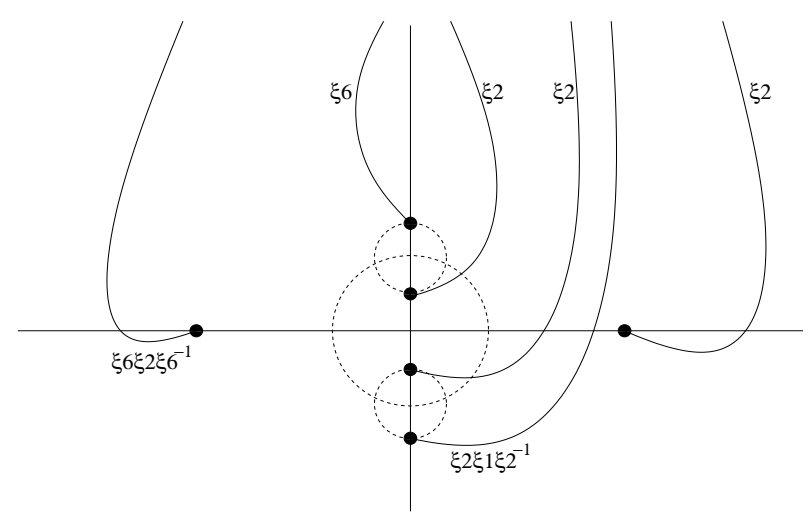

Figure 15. Generators at $x=\eta_{4}-\varepsilon$ 
Monodromy relations at $x=\eta_{5}$. At $x=\eta_{4}-\varepsilon$, the generators are as in Figure 15. Now, when $x$ makes half-turn counter-clockwise on the circle $\mathbb{S}_{\varepsilon}\left(\eta_{4}\right)$, from $\eta_{4}-\varepsilon$ to $\eta_{4}+\varepsilon$, then moves on the real axis from $\eta_{4}+\varepsilon$ to $\eta_{5}-\varepsilon$, the $\xi_{k}$ 's are deformed as in Figure 16, where

$$
\begin{aligned}
\alpha & :=\left(\xi_{2}^{-1} \xi_{6} \xi_{2}\right)^{-1} \cdot \xi_{6} \xi_{2} \xi_{6}^{-1} \cdot\left(\xi_{2}^{-1} \xi_{6} \xi_{2}\right), \\
\beta & :=\left(\xi_{1} \xi_{2}^{-1}\right)^{-1} \cdot\left(\xi_{2} \xi_{1}\right) \cdot \xi_{2} \cdot\left(\xi_{2} \xi_{1}\right)^{-1} \cdot\left(\xi_{1} \xi_{2}^{-1}\right) .
\end{aligned}
$$

The singular line $L_{\eta_{1}}$ is tangent to the curve at two simple points, in both cases with intersection multiplicity 2 . The monodromy relations at $x=\eta_{5}$ are then given as follows:

$$
\begin{aligned}
& \left(\xi_{1} \xi_{2}^{-1}\right)^{-1} \cdot \xi_{2} \cdot\left(\xi_{1} \xi_{2}^{-1}\right)=\left(\xi_{2} \xi_{1}\right)^{-1} \cdot \xi_{1} \cdot\left(\xi_{2} \xi_{1}\right) \\
& \left(\xi_{6} \xi_{2} \xi_{6}^{-1}\right) \cdot \xi_{2} \cdot\left(\xi_{6} \xi_{2} \xi_{6}^{-1}\right)^{-1}=\xi_{2}^{-1} \xi_{6} \xi_{2} .
\end{aligned}
$$

By (4.4) and (4.12), both of them are equivalent to

$$
\xi_{2} \xi_{1} \xi_{1}=\xi_{1} \xi_{1} \xi_{2}
$$

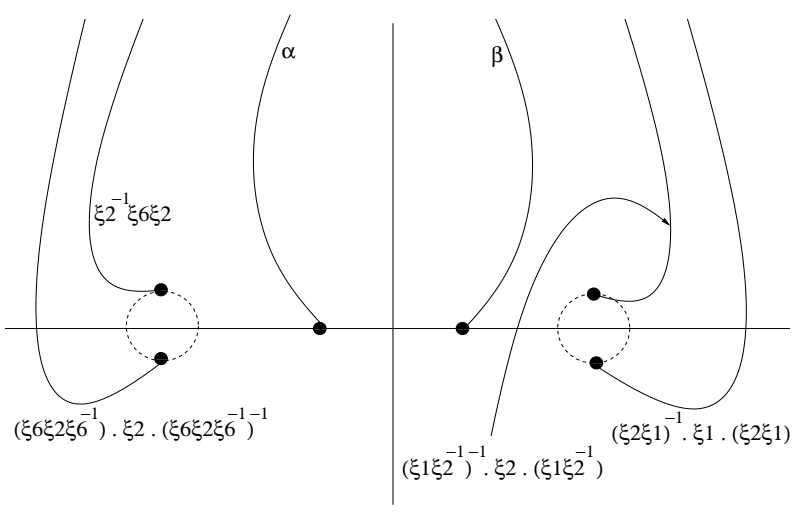

FIGURE 16. Generators at $x=\eta_{5}-\varepsilon$

Monodromy relations at $x=\eta_{6}$. In Figure 17, we show how the generators are deformed when $x$ makes half-turn counter-clockwise on the circle $\mathbb{S}_{\varepsilon}\left(\eta_{5}\right)$, from $\eta_{5}-\varepsilon$ to $\eta_{5}+\varepsilon$, and then moves on the real axis from $\eta_{5}+\varepsilon$ to $\eta_{6}-\varepsilon$. The singular line $L_{\eta_{6}}$ passes through the points $(1,1)$ and $(1,-1)$ which are both singularities of type $\mathbf{A}_{4}$. Puiseux parametrizations of the curve at these points are given by:

$$
\begin{aligned}
(1,1): & x=1+t^{2}, \quad y=1+t^{4}+3 i \sqrt{3} t^{5}+\text { higher terms } \\
(1,-1): & x=1+t^{2}, \quad y=-1-t^{4}-3 i \sqrt{3} t^{5}+\text { higher terms. }
\end{aligned}
$$

The monodromy relations at $x=\eta_{6}$ are then given by

$$
\begin{aligned}
& \xi_{2} \cdot \xi_{1} \xi_{2} \xi_{1}^{-1} \cdot \xi_{2} \cdot \xi_{1} \xi_{2} \xi_{1}^{-1} \cdot \xi_{2}=\xi_{1} \xi_{2} \xi_{1}^{-1} \cdot \xi_{2} \cdot \xi_{1} \xi_{2} \xi_{1}^{-1} \cdot \xi_{2} \cdot \xi_{1} \xi_{2} \xi_{1}^{-1} \\
& \alpha=\left(\xi_{2}^{-1} \xi_{6} \xi_{2} \alpha\right)^{2} \cdot \xi_{2}^{-1} \xi_{6} \xi_{2} \cdot\left(\xi_{2}^{-1} \xi_{6} \xi_{2} \alpha\right)^{-2} .
\end{aligned}
$$


Notice that (4.16) is automatically satisfied, while (4.17) can be written as

$$
\left(\xi_{2} \xi_{1}\right)^{4}=\xi_{1} \xi_{2}
$$

or, equivalently, as

$$
\left(\xi_{2} \xi_{1} \xi_{1}\right)^{2}=e .
$$

Indeed, using (4.15) under the form $\xi_{1}^{-1} \xi_{2} \xi_{1}=\xi_{1} \xi_{2} \xi_{1}^{-1}$, the relation (4.16) can be written as

$$
\xi_{2} \cdot\left(\xi_{1}^{-1} \xi_{2} \xi_{1} \cdot \xi_{2} \cdot \xi_{1}\right) \xi_{2} \xi_{1}^{-1} \cdot \xi_{2}=\xi_{1}^{-1} \xi_{2} \xi_{1} \cdot \xi_{2} \cdot\left(\xi_{1}^{-1} \xi_{2} \xi_{1} \cdot \xi_{2} \cdot \xi_{1}\right) \xi_{2} \xi_{1}^{-1},
$$

which is nothing but $\xi_{1} \xi_{2} \cdot \xi_{2} \xi_{1} \xi_{2}=\xi_{2} \xi_{1} \cdot \xi_{2} \xi_{2} \xi_{1}$ by (4.12). By (4.5) this equality is always satisfied. The relation (4.17) is written as

$$
\xi_{6} \xi_{2} \xi_{6}^{-1} \cdot\left(\xi_{2}^{-1} \xi_{6} \xi_{2} \cdot \xi_{6} \xi_{2} \xi_{6}^{-1}\right)^{2}=\left(\xi_{2}^{-1} \xi_{6} \xi_{2} \cdot \xi_{6} \xi_{2} \xi_{6}^{-1}\right)^{2} \cdot \xi_{2}^{-1} \xi_{6} \xi_{2},
$$

which is the same as

$$
\xi_{6} \xi_{2} \xi_{6}^{-1} \cdot\left(\xi_{6} \xi_{2} \xi_{6}^{-1} \cdot \xi_{2}\right)^{2}=\left(\xi_{6} \xi_{2} \xi_{6}^{-1} \cdot \xi_{2}\right)^{2} \cdot \xi_{2}^{-1} \xi_{6} \xi_{2}
$$

by (4.14). Equivalently $\xi_{6} \xi_{2} \xi_{6}^{-1} \cdot \xi_{2} \cdot \xi_{6} \xi_{2} \xi_{6}^{-1}=\xi_{2} \xi_{6} \xi_{2}$. By (4.4), $\xi_{6}$ can be eliminated so

$$
\xi_{1} \xi_{2} \cdot\left(\xi_{2} \xi_{1}\right)^{-1} \cdot \xi_{2} \xi_{2} \xi_{1}=\xi_{2} \xi_{1} \xi_{2} \xi_{1} \xi_{2}^{-1} .
$$

By (4.5) this is the same as $\xi_{1} \xi_{2}=\left(\xi_{2} \xi_{1}\right)^{4}$, while (4.5) and (4.12) show that (4.201) is also the same as $\left(\xi_{2} \xi_{1} \xi_{1}\right)^{2}=e$.

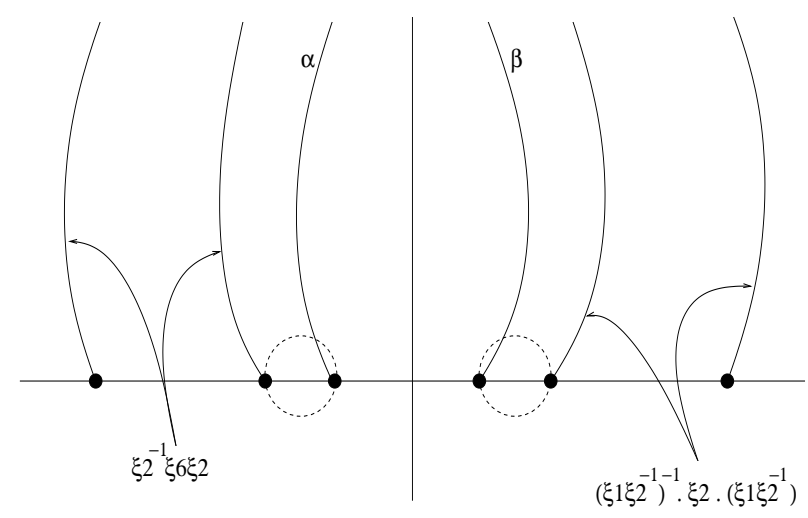

FiguRE 17. Generators at $x=\eta_{6}-\varepsilon$

It remains to find the monodromy relations around the singular lines $L_{\eta_{7}}$ and $L_{\eta_{8}}$ corresponding to the non-real roots $\eta_{7}$ and $\eta_{8}$ of the discriminant $\Delta_{y}(f)(x)$. 
Monodromy relations at $x=\eta_{7}$ and $x=\eta_{8}$. Figure 17 shows the generators at $x=\eta_{6}-\varepsilon$. In Figure 18 (respectively Figure 19), we show how the $\xi_{k}$ 's are deformed when $x$ makes half-turn counter-clockwise on the circle $\mathbb{S}_{\varepsilon}\left(\eta_{6}\right)$, from $\eta_{6}-\varepsilon$ to $\eta_{6}+\varepsilon$, then moves on the real axis from $\eta_{6}+\varepsilon$ to $\Re\left(\eta_{7}\right)$, and finally moves straight along the line $\left(\Re\left(\eta_{7}\right), \eta_{7}\right)$ from $\Re\left(\eta_{7}\right)$ to $\Re\left(\eta_{7}\right)+i\left(\Im\left(\eta_{7}\right)+\varepsilon\right)$ (respectively along the line $\left(\Re\left(\eta_{8}\right), \eta_{8}\right)$ from $\Re\left(\eta_{8}\right)$ to $\left.\Re\left(\eta_{8}\right)+i\left(\Im\left(\eta_{8}\right)-\varepsilon\right)\right)$, where

$$
\gamma:=\left(\xi_{2}^{-1} \xi_{6} \xi_{2} \alpha\right)^{-1} \cdot \alpha \cdot\left(\xi_{2}^{-1} \xi_{6} \xi_{2} \alpha\right)
$$

(In these figures we concentrate only on the generators which may give a priori some relations.) The monodromy relations at $x=\eta_{7}$ and $x=\eta_{8}$ are multiplicity 2 tangent relations given by

$$
\gamma=\xi_{2}
$$

and

$$
(\alpha \gamma)^{-1} \cdot \xi_{2}^{-1} \xi_{6} \xi_{2} \cdot(\alpha \gamma)=\xi_{1}^{-1} \xi_{2} \xi_{1}
$$

respectively. In fact, these relations are automatically satisfied. Indeed, the relation (4.21) is written as

$$
\xi_{6} \xi_{2} \xi_{6}^{-1} \cdot\left(\xi_{2}^{-1} \xi_{6} \xi_{2} \cdot \xi_{6} \xi_{2} \xi_{6}^{-1}\right) \cdot \xi_{2}^{-1} \xi_{6}=\left(\xi_{2}^{-1} \xi_{6} \xi_{2} \cdot \xi_{6} \xi_{2} \xi_{6}^{-1}\right) \cdot \xi_{2}^{-1} \xi_{6} \xi_{2} .
$$

But, by (4.14), we know that $\xi_{2}^{-1} \xi_{6} \xi_{2} \cdot \xi_{6} \xi_{2} \xi_{6}^{-1}=\xi_{6} \xi_{2} \xi_{6}^{-1} \cdot \xi_{2}$, so the relation above is always satisfied. Now, using (4.21), the relation (4.22) is written as

$$
\left(\xi_{2}^{-1} \xi_{6} \xi_{2} \cdot \xi_{6} \xi_{2} \xi_{6}^{-1}\right) \cdot \xi_{2}^{-1} \xi_{6} \xi_{2} \cdot \xi_{2}=\xi_{6} \xi_{2} \xi_{6}^{-1} \cdot \xi_{2}^{-1} \xi_{6} \xi_{2} \cdot \xi_{2} \cdot \xi_{1}^{-1} \xi_{2} \xi_{1},
$$

which is equivalent to $\xi_{2} \xi_{6} \xi_{2} \xi_{2}=\xi_{6} \xi_{2} \xi_{2} \xi_{1}^{-1} \xi_{2} \xi_{1}$ by (4.14). The latter is always satisfied, by (4.4).

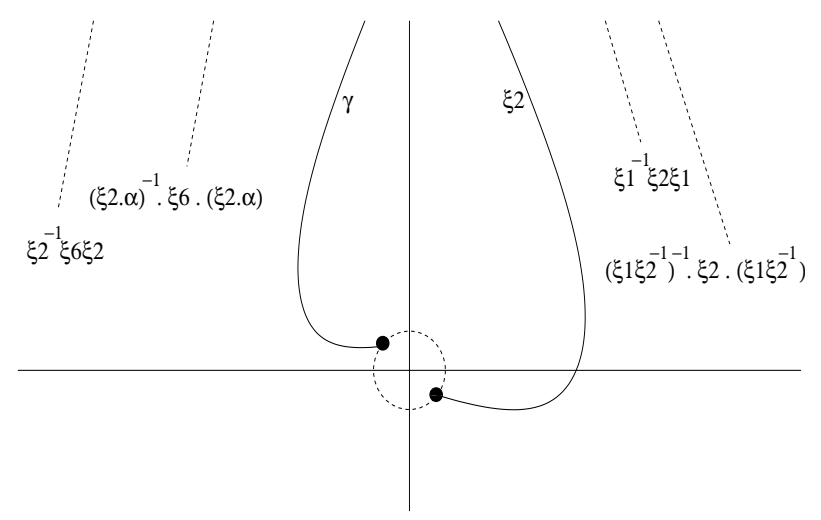

FiguRE 18. Generators at $x=\Re\left(\eta_{7}\right)+i\left(\Im\left(\eta_{7}\right)+\varepsilon\right)$ 


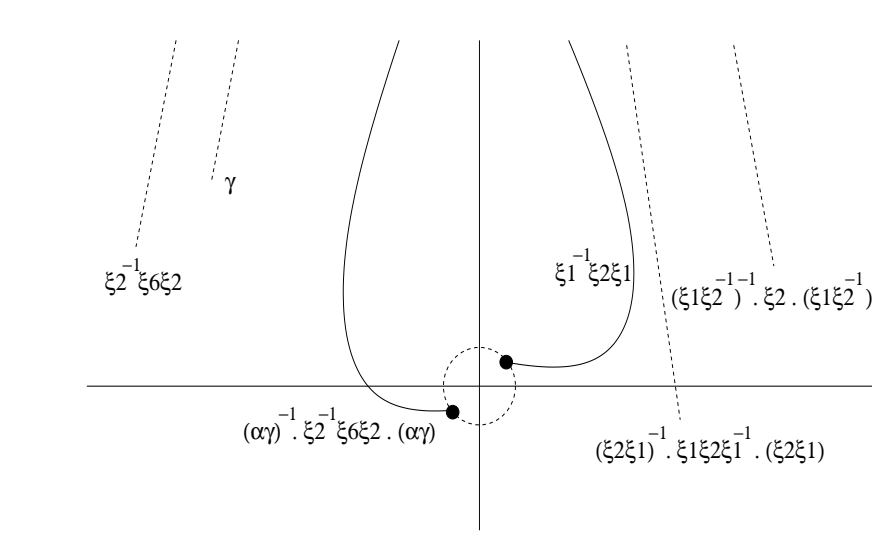

FIGURE 19. Generators at $x=\Re\left(\eta_{8}\right)+i\left(\Im\left(\eta_{8}\right)-\varepsilon\right)$

Altogether, we have proved that the fundamental group $\pi_{1}\left(\mathbb{C P}^{2} \backslash C^{\prime}\right)$ is presented by the generators $\xi_{1}$ and $\xi_{2}$ and the relations (4.5), (4.12), (4.15) and (4.18). The relation (4.5) can be written as

$$
\left(\xi_{2} \xi_{1} \xi_{2}\right)^{2}=e
$$

This shows that (4.12) is equivalent to (4.18). The relation (4.15) is automatically satisfied. Indeed, by (4.18), it is equivalent to

$$
\left(\xi_{2} \xi_{1} \xi_{1}\right)^{2}=\left(\xi_{1} \xi_{2} \xi_{1}\right)^{2}
$$

But both sides are equal to $e$, by (4.18) under the form (4.19). Hence, $\pi_{1}\left(\mathbb{C P}^{2} \backslash C^{\prime}\right)$ is simply presented by the generators $\xi_{1}$ and $\xi_{2}$ and the relations (4.18) and (4.23). After the change $a:=\xi_{2} \xi_{1} \xi_{2}$ and $b:=\xi_{2} \xi_{1}$, the presentation is also given by

$$
\pi_{1}\left(\mathbb{C P}^{2} \backslash C^{\prime}\right) \simeq\left\langle a, b \mid a^{2}=e, a b a=b^{4}\right\rangle .
$$

Lemma 4.1. The generator b satisfies the following two properties:

(1) $b^{15}=e$;

(2) $b^{5}$ is in the centre of $\pi_{1}\left(\mathbb{C P}^{2} \backslash C^{\prime}\right)$.

Proof. Since $a^{2}=e$, the relation $a b a=b^{4}$ gives $b^{16}=a b^{4} a=b$, that is, $b^{15}=e$ as desired. To show that $b^{5}$ is in the centre of $\pi_{1}\left(\mathbb{C P}^{2} \backslash C^{\prime}\right)$ we write:

$$
\begin{array}{r}
b^{5} a b^{-5} a^{-1}=b \cdot b^{4} \cdot a b^{-5} a^{-1}=b \cdot a b a \cdot a b^{-5} a^{-1}= \\
b a \cdot b^{-4} \cdot a^{-1}=b a \cdot a^{-1} b^{-1} a^{-1} \cdot a^{-1}=e .
\end{array}
$$


It follows from the lemma that $\pi_{1}\left(\mathbb{C P}^{2} \backslash C^{\prime}\right)$ is also presented as:

$$
\begin{aligned}
\pi_{1}\left(\mathbb{C P}^{2} \backslash C^{\prime}\right) & \simeq\left\langle a, b \mid a^{2}=e, a b a=b^{4}, b^{15}=e, b^{5} a=a b^{5}\right\rangle \\
& \simeq\langle a, b, c, d| a^{2}=b^{15}=e, a b a=b^{4}, b^{5} a=a b^{5}, c=b^{6}, \\
& \left.\quad d=b^{5}, d a=a d, d b=b d, d c=c d\right\rangle \\
& \simeq\langle a, b, c, d| a^{2}=b^{15}=e, a b a=b^{4}, c=b^{6}, d=b^{5}, \\
& \left.\quad b=c d^{-1}, d a=a d, d b=b d, d c=c d\right\rangle \\
& \simeq\left\langle a, c, d \mid a^{2}=c^{5}=d^{3}=e, a c d^{-1} a=c^{4} d^{-1}, d a=a d, d c=c d\right\rangle \\
& \simeq\left\langle a, c, d \mid a^{2}=c^{5}=d^{3}=e, a c a=c^{4}, d a=a d, d c=c d\right\rangle \\
& \simeq \mathbb{D}_{10} \times(\mathbb{Z} / 3 \mathbb{Z}) .
\end{aligned}
$$

\section{REFERENCES}

1. E. Artal Bartolo, Sur les couples de Zariski, J. Algebraic Geom. 3 (1994) 223-247.

2. E. Artal Bartolo and J. Carmona Ruber, Zariski pairs, fundamental groups and Alexander polynomials, J. Math. Soc. Japan 50 (1998) 521-543.

3. A. Degtyarev, Oka's conjecture on irreducible plane sextics, J. London Math. Soc. (to appear).

4. A. Degtyarev, On irreducible sextics with non-abelian fundamental group, Proceedings of Niigata-Toyama Conferences 2007, Adv. Stud. Pure Math. (to appear).

5. C. Eyral and M. Oka, A proof of a conjecture of Degtyarev on non-torus plane sextics, Proceedings of Niigata-Toyama Conferences 2007, Adv. Stud. Pure Math. (to appear).

6. Lê D. T. and C. P. Ramanujam, The invariance of Milnor number implies the invariance of the topological type, Amer. J. Math. 98 (1976) 67-78.

7. M. Oka, Symmetric plane curves with nodes and cusps, J. Math. Soc. Japan 44 (3) (1992) $375-414$.

8. M. Oka, Flex curves and their applications, Geometriae Dedicata 75 (1999) 67-100.

9. M. Oka, A new Alexander-equivalent Zariski pair, Acta Math. Vietnam. 27 (3) (2002) 349-357.

10. M. Oka and D. T. Pho, Classification of sextics of torus type, Tokyo J. Math. 25 (2) (2002) 399-433.

11. D. T. Pho, Classification of singularities on torus curves of type $(2,3)$, Kodai Math. J. 24 (2001) 259-284.

12. E. R. van Kampen, On the fundamental group of an algebraic curve, Amer. J. Math. $\mathbf{5 5}$ (1933) 255-260.

13. O. Zariski, On the problem of existence of algebraic functions of two variables possessing a given branch curve, Amer. J. Math. 51 (1929) 305-328.

14. O. Zariski, On the irregularity of cyclic multiple planes, Ann. of Math. 32 (1931) 445-489.

15. O. Zariski, The topological discriminant group of a Riemann surface of genus $p$, Amer. J. Math. 59 (1937) 335-358.

16. O. Zariski, Studies in equisingularity II. Equisingularity in codimension 1 (and characteristic zero), Amer. J. Math. 87 (1965) 972-1006.

17. O. Zariski, Contribution to the problem of equisingularity, Questions on Algebraic Varieties (C.I.M.E., III Ciclo, Varenna, 1969), Ed. Cremonese, Roma (1970), 261-343. 
Department of Mathematical Sciences, University of Aarhus, Building 1530, Ny Munkegade, DK-8000 Aarhus C, Denmark

E-mail address: eyralchr@yahoo.com

Department of Mathematics, Tokyo University of Science, 26 Wakamiya-cho, SHINJUKU-KU, TOKYO 162-8601, JAPAN

E-mail address: oka@rs.kagu.tus.ac.jp 